\title{
Less Invasive Ablation of Atrial Fibrillation Achieved by Contrast-Free Cryoballoon Ablation
}

\author{
Hiroshi Miyama, MD; Seiji Takatsuki, MD, PhD
}

C ryoballoon ablation (CBA) has become one of the most frequently used techniques for ablation of atrial fibrillation (AF). The pulmonary veins (PVs) are isolated effectively and efficiently by a single-shot cryoenergy application, which has been shown to be non-inferior to conventional radiofrequency ablation (RFA) in terms of efficacy and safety, and the total procedure time has been shortened. ${ }^{1}$ Furthermore, another advantage of $\mathrm{CBA}$ is that the learning curve is short and the procedural success is less operator-dependent. ${ }^{2}$ Because of its high efficacy and versatility, CBA has become the mainstream

\section{Article p 256}

procedure for $\mathrm{AF}$ ablation, and several tips and techniques to improve performance have been reported (Figure). ${ }^{36}$ There are also plenty of tips for avoiding complications associated with CBA (i.e., PV stenosis and phrenic nerve injury (PNI). ${ }^{7,8}$ In particular, as compared with RFA, which can be accomplished contrast-free by using a 3D mapping system, CBA usually requires a step with contrast infusion from the distal tip of the balloon catheter to
Improving performance

(1) Hockey stick technique

Sheath curved in a hockey stick figure to fit inf. PVs (3).

(2) Pull-down technique

Pulling back balloon to occlude inf. gap during freezing (3).

\section{(3) $\boldsymbol{R} V$ rapid pacing}

Facilitating freezing during inadequate balloon cooling (4).

(4) Time to isolation based ablation Set duration of cryo-application 1 min after PV isolation (5).

(5) LA roof/bottom linear ablation Procedure for LA posterior wall isolation (6).

\section{Tips for Cryoablation}
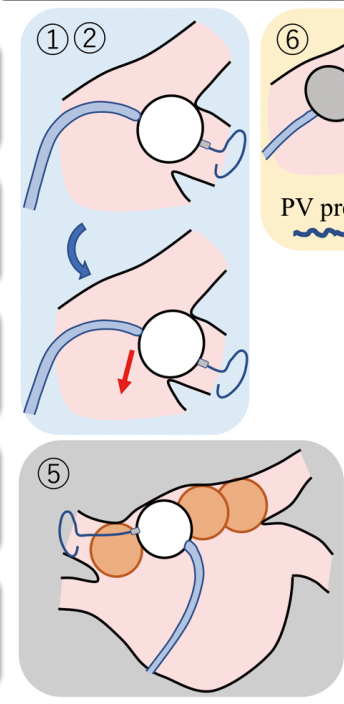

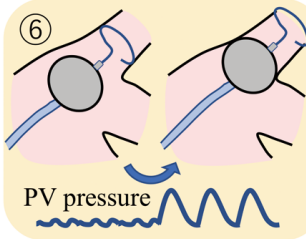

(8)

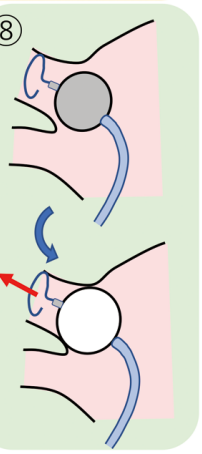

\section{Avoiding complications}

\section{(6) PV pressure monitoring}

Wedge pressure of distal tip get higher by PV occlusion (11).

\section{(7) ICE guidance}

PV occlusion confirmed by ICE $(9,12)$.

(8) Proximal-seal technique Sealing PV after freezing to avoid distal cryo-application.

\section{(9) Phrenic nerve pacing}

To avoid PNI with monitoring

CMAP during right PV ablation (7).

Figure. Tips for successful cryoballoon ablation. Tips for improving procedural performance (Left), and for avoiding complications (Right). Each gray and white balloon indicates the status before and during freezing. Red arrows indicate the direction of the torque. CMAP, compound motor action potential; ICE, intracardiac echocardiography; LA, left atrium; PNI, phrenic nerve injury; $\mathrm{PV}$, pulmonary vein; RV, right ventricle. The numbers in the brackets means the reference numbers.

The opinions expressed in this article are not necessarily those of the editors or of the Japanese Circulation Society.

Received July 29, 2021; accepted July 29, 2021; J-STAGE Advance Publication released online September 7, 2021

Department of Cardiology, Keio University School of Medicine, Tokyo, Japan

Mailing address: Seiji Takatsuki, MD, PhD, Department of Cardiology, Keio University School of Medicine, 35 Shinanomachi, Shinjuku-ku, Tokyo 160-8582, Japan. E-mail: seiji.takatsuki@gmail.com

All rights are reserved to the Japanese Circulation Society. For permissions, please e-mail: cj@j-circ.or.jp ISSN-1346-9843 
confirm occlusion of the PVs with the balloon before cryoapplications. This process is one of the important limitations of CBA and mostly concerns patients with renal dysfunction or allergies to contrast media.

In this issue of the Journal, Suzuki et al ${ }^{9}$ show a practical and quantitative method for confirming balloon fitting to the PVs by using intracardiac echocardiography (ICE) during the procedure. This ICE-based assessment, which has the potential to overcome the limitations of CBA, is a further less invasive ablation strategy for AF patients.

In order to perform CBA without contrast injection, the appropriate balloon-contact with the PVs can be confirmed by tactile feedback, ${ }^{10}$ balloon motion under fluoroscopy, and pressure waveforms acquired from the distal tip of the catheter;"11 however, all those methods lack quantification. Although confirming peri-balloon leakage with ICE and adjusting the balloon-position has been reported recently, the goal is set as complete disruption of the PV leak flow (i.e., a total PV-occlusion), and there has been less study of peri-balloon leak flow velocity (PVLF). ${ }^{12}$

In the first phase of the present investigation, peri-balloon leakage was compared between ICE and conventional contrast infusion. PVLF was assessed by ICE and the electrical PV lesion gaps were evaluated by a high-density electroanatomical mapping system. Although the presence of PV leakage assessed by ICE did not always result in failure of PV isolation, a correlation was shown between higher PVLF and smaller electrical PV lesion gaps, which can be naturally understood from the relationship of blood flow velocity and the blood flow passing area. Interestingly, PV leakage was confirmed more often with conventional contrast infusion that with an ICE-guided evaluation and was not always consistent. This could be explained by overestimation of PV leakage due to the excessive PV pressure caused by contrast infusion, which may incite unnecessary pushing of the balloon on the PVs. ICE-guided CBA enables more accurate and quantitative assessment of PV leakage, and reduces the risk of excessive balloon contact with the PVs inducing stenosis and PNI. ${ }^{13-15}$

In the second phase, efficacy was compared between the conventional contrast-guided method and the ICE-guided protocol based on the ideal PVLF analyzed from the firstphase analyses. In the ICE-guided group, PV isolation was successfully achieved in all cases without a "rescue" contrast injection, and there was no difference in the recurrence rate compared with the conventional protocol group at 6 months after the procedure without increasing the complications. This study showed the feasibility and efficacy of ICE-guided contrast-free CBA, and moreover, less invasiveness of the procedure not only in terms of renoprotection but also for avoiding complications related to the ablation procedure itself (i.e., PV stenosis, PNI). In the future, it will be necessary to confirm the versatility of this ICE-guided procedure by other operators and to ensure the accuracy of PVLF evaluation by conducting multicenter randomized controlled trials.

\section{Acknowledgment}

We thank Mr. John Martin for his linguistic advice.

\section{Disclosures}

Dr. Takatsuki received an unrestricted research grant from Japan
Lifeline and personal fees from Medtronic and Daiichisankyo. The other author reports no conflicts of interest.

\section{References}

1. Kuck KH, Brugada J, Furnkranz A, Metzner A, Ouyang F, Chun $\mathrm{KR}$, et al. Cryoballoon or radiofrequency ablation for paroxysmal atrial fibrillation. N Engl J Med 2016; 374: 2235-2245.

2. Providencia R, Defaye P, Lambiase PD, Pavin D, Cebron JP, Halimi F, et al. Results from a multicentre comparison of cryoballoon vs. radiofrequency ablation for paroxysmal atrial fibrillation: Is cryoablation more reproducible? Europace 2017; 19: $48-57$.

3. Chen S, Schmidt B, Bordignon S, Bologna F, Nagase T, Perrotta $\mathrm{L}$, et al. Practical techniques in cryoballoon ablation: How to isolate inferior pulmonary veins. Arrhythm Electrophysiol Rev 2018; 7: 11-17.

4. Nishimura T, Okishige K, Yamauchi Y, Shigeta T, Nakamura $\mathrm{R}$, Hirao $\mathrm{T}$, et al. Clinical impact of rapid ventricular pacing on the left atrial posterior wall isolation by a cryoballoon application: A randomized controlled trial. J Interv Card Electrophysiol 2020; 59: 565-573.

5. Aryana A, Kenigsberg DN, Kowalski M, Koo CH, Lim HW, O'Neill PG, et al. Verification of a novel atrial fibrillation cryoablation dosing algorithm guided by time-to-pulmonary vein isolation: Results from the Cryo-DOSING Study (Cryoballoonablation DOSING Based on the Assessment of Time-to-Effect and Pulmonary Vein Isolation Guidance). Heart Rhythm 2017; 14: $1319-1325$.

6. Aryana A, Allen SL, Pujara DK, Bowers MR, O'Neill PG, Yamauchi Y, et al. Concomitant pulmonary vein and posterior wall isolation using cryoballoon with adjunct radiofrequency in persistent atrial fibrillation. JACC Clin Electrophysiol 2021; 7: 187-196.

7. Casado-Arroyo R, Chierchia GB, Conte G, Levinstein M, Sieira $\mathrm{J}$, Rodriguez-Manero M, et al. Phrenic nerve paralysis during cryoballoon ablation for atrial fibrillation: A comparison between the first- and second-generation balloon. Heart Rhythm 2013; 10: $1318-1324$.

8. Okishige K, Aoyagi H, Kawaguchi N, Katoh N, Yamashita M, Nakamura T, et al. Novel method for earlier detection of phrenic nerve injury during cryoballoon applications for electrical isolation of pulmonary veins in patients with atrial fibrillation. Heart Rhythm 2016; 13: 1810-1816.

9. Suzuki A, Fujiwara R, Asada H, Iwasa K, Miyata T, Song WH, et al. Peri-balloon leak flow velocity assessed by intra-cardiac echography predicts pulmonary vein electrical gap: Intra-cardiac echography-guided contrast-free cryoballoon ablation. Circ $J$ 2022; 86: $256-265$.

10. Kühne M, Knecht S, Spies F, Aeschbacher S, Haaf P, Zellweger $\mathrm{M}$, et al. Cryoballoon ablation of atrial fibrillation without demonstration of pulmonary vein occlusion: The Simplify Cryo Study. Front Cardiovasc Med 2021; 8: 664538.

11. Hasegawa K, Miyazaki S, Kaseno K, Hisazaki K, Amaya N, Miyahara K, et al. Pressure-guided second-generation cryoballoon pulmonary vein isolation: Prospective comparison of the procedural and clinical outcomes with the conventional strategy. J Cardiovasc Electrophysiol 2019; 30: 1841-1847.

12. Maalouf J, Whiteside HL, Pillai A, Omar A, Berman A, Saba S, et al. Reduction of radiation and contrast agent exposure in a cryoballoon ablation procedure with integration of electromagnetic mapping and intracardiac echocardiography: A single center experience. J Interv Card Electrophysiol 2020; 59: 545550 .

13. Tokutake K, Tokuda M, Yamashita S, Sato H, Ikewaki H, Okajima E, et al. Anatomical and procedural factors of severe pulmonary vein stenosis after cryoballoon pulmonary vein ablation. JACC Clin Electrophysiol 2019; 5: 1303-1315.

14. Miyama H, Takatsuki S, Hashimoto K, Yamashita T, Fujisawa $\mathrm{T}$, Katsumata Y, et al. Anatomical changes in the pulmonary veins and left atrium after cryoballoon ablation. Pacing Clin Electrophysiol 2020; 43: 1289-1294.

15. Saitoh Y, Ströker E, Irfan G, Mugnai G, Ciconte G, Hünük B, et al. Fluoroscopic position of the second-generation cryoballoon during ablation in the right superior pulmonary vein as a predictor of phrenic nerve injury. Europace 2016; 18: 1179-1186. 